\title{
Upper Tract Transitional Carcinoma: An Array of Imaging Conundrum
}

Raihanah Haroon, Siti Kamariah Che Mohamed, Khairunisa Ahmad Affandi

International Islamic University Malaysia

Case report: We report a 38-year-old gentleman who presented with painless haematuria for 6-months duration. Contrast-enhanced CT renal revealed a large, calcified intrapelvic mass with calyceal extension confined within the right kidney with moderate pelvicalyceal dilatation but no evidence of synchronous lesion elsewhere. Histopathological analysis showed high grade urothelial carcinoma of the renal pelvis with invasion of the renal parenchyma. Upper tract urothelial carcinoma (UTUC) which was once thought similar to urothelial carcinoma of the bladder (UCB), is increasingly being recognized as a different entity. However, heterogenous array of imaging manifestations of UTUC is also increasingly being encountered and pose diagnostic challenges though UTUC is not as prevalent as UCB which is easier to diagnose. Despite distinctive features against renal cell carcinoma (RCC), UTUC may mimic numerous other renal pathology especially the benign ones hence imaging plays a pivotal role to ascertain the diagnosis. This disease is usually treated with radical nephroureterectomy with superadded neoadjuvant chemotherapy for high risk cases. Less invasive endoscopic resection is also offered in certain cases but stringent surveillance of the whole urinary tract as well as post-operative follow-up are mandatory. 